\title{
Pediatric low-grade glioma
}

\author{
Anita Mahajan
}

Received: 21 October 2012 / Accepted: 6 November 2012 /Published online: 19 November 2012

(C) Springer-Verlag Berlin Heidelberg 2012

\begin{abstract}
Childhood brain tumors constitute $20-25 \%$ of all childhood cancers diagnosed in the USA with the most common central nervous system (CNS) tumors being astrocytoma, medulloblastoma, and ependymoma. Glial tumors make up $56 \%$ of all CNS tumors in children under the age of 14 and $45 \%$ of all CNS tumors in adolescents. In both age groups, gliomas represent two thirds of all CNS tumors. Low-grade gliomas (LGG) include a heterogeneous group of tumors that not only have some common features, but also have characteristics that can be important for management decisions. It is important to have a multidisciplinary approach in the diagnosis and management of these young patients since most will be expected to have a long-term survival. In this article, the characteristics, diagnosis, management options, and outcomes for pediatric LGG will be reviewed.
\end{abstract}

Keywords Low-grade glioma · Pilocytic astrocytoma ·

Radiotherapy $\cdot$ Chemotherapy

\section{Introduction}

As reported by the Central Brain Tumor Registry of the United States, central nervous system (CNS) tumors are the second most common neoplasm diagnosed in children, constituting $20-25 \%$ of all childhood cancers in the USA with an incidence of 5.05 cases $/ 10^{5}$ person-years. In the USA, 20,273 CNS tumors were diagnosed between 2004 and 2008, representing approximately $7 \%$ of all CNS tumors diagnosed. For children aged 0-14 years, the most

A. Mahajan $(\bowtie)$

Department of Radiation Oncology,

UT MD Anderson Cancer Center, 1515 Holcombe Boulevard,

Houston, TX 77005, USA

e-mail: amahajan@mdanderson.org common histologies diagnosed were pilocytic astrocytoma (PA), embryonal tumors, and malignant glioma. In the older group, 15-19 years, the most common tumors are pituitary tumors and PA. Overall, within the pediatric population, glial tumors constituted the most common histology in the $0-14$-year and 15-19-year age groups (56 and $45 \%$, respectively) [1].

According to the 2007 World Health Organization classification system, neuroepithelial tumors, and specifically glial tumors, encompass a variety of subtypes including astrocytic, oligodendroglial, oligoastrocytic, ependymal, and neuroglial tumors. The first four histologic subtypes make up $70 \%$ of the CNS tumors, and of these, $80-90 \%$ are low-grade astrocytomas [2]. In this review, the diagnosis, management, and outcomes of non-ependymal low-grade gliomas (LGGs) will be reviewed.

\section{Presentation and diagnosis}

Patients diagnosed with LGGs present with a variety of signs and symptoms depending on the age of the patient and the tumor size and location. It is not unusual for a LGG to be diagnosed incidentally on an imaging study done for an unassociated reason. Symptoms may have been present for several months since these tumors tend to enlarge slowly over time. The two most common sites of origin for LGGs are in the posterior fossa and within the cerebellar hemisphere, in the fourth ventricle, in the hypothalamic area, or optic pathways. For those that arise in the posterior fossa, patients may present with headaches, nausea, vomiting, double vision, or gait imbalance. On evaluation, they may be found to have papilledema, cranial nerve palsies, cerebellar findings, and lateralized weakness. Patients with hypothalamic tumors may present with visual changes, delayed growth, precocious puberty, or symptoms of increased 
intracranial pressure. Very young children with large hypothalamic LGGs may present with failure to thrive, otherwise known as the diencephalic syndrome. For patients with supratentorial hemispheric tumors or thalamic tumors, symptoms vary depending on the local disruption of normal function. Seizure activity is a common presenting finding of tumors located within the temporal lobes or with compression of the cerebral cortex.

The typical evaluation and work-up for a patient with a suspected brain tumor is a good clinical evaluation to assess the overall status of the patient and to identify any symptoms that may suggest a particular tumor location. Brain imaging is necessary. Computerized tomography (CT) scanning with and without contrast is a test that is usually readily available and can rule out critical situations such as a significant hemorrhage, hydrocephalus, or significant mass effect that require emergent surgical evaluation and possible intervention. In all cases, a magnetic resonance imaging (MRI) should be performed prior to surgical intervention if possible to further characterize the tumor. CT and MRI may give some indications about the nature of the tumor by the location, growth pattern, enhancement pattern, and imaging characteristics of the primary tumor. These imaging modalities are important to determine whether there are other sites of disease and the presence of other intracranial abnormalities that could support other diagnoses such as neurofibromatosis (NF1 or NF2).

MRI characteristics can correlate with histopathologic diagnosis. Examples of MRI scans of typical LGG are shown in Fig. 1. The enhancement pattern, the presence or absence of a distinct tumor margin, and invasiveness may suggest a particular diagnosis. Forbes et al. reported that a relatively higher diffusion-weighted imaging signal is associated with high-grade posterior fossa tumors such as medulloblastoma rather than LGG [3]. Grade 1 gliomas are usually associated with contrast uptake, whereas grade 2 gliomas usually do not take up contrast. Higher grade tumors tend to be associated with enhancement. MR spectroscopy may also help in separating higher and lower grade tumors.

The differential diagnosis varies depending on the tumor location for supratentorial lesions. In the hypothalamic and pineal areas, serum beta-HCG and alpha-fetoprotein evaluation may be useful in distinguishing germ cell tumors
Fig. 1 a Axial T2-weighted and $\mathbf{b} \mathrm{T} 1$ contrast-enhanced MRI scans of a patient with a pilocytic astrocytoma within the brainstem. Note the wellcircumscribed mass with heterogeneous enhancement and minimal edema. c Axial T1 contrast-enhanced and $\mathbf{d}$ coronal T2-weighted MRI scans illustrating a large wellcircumscribed non-enhancing thalamic pilocytic astrocytoma in an 18-month-old patient presenting with failure to thrive. Note the mass effect on the hypothalamus and midline shift
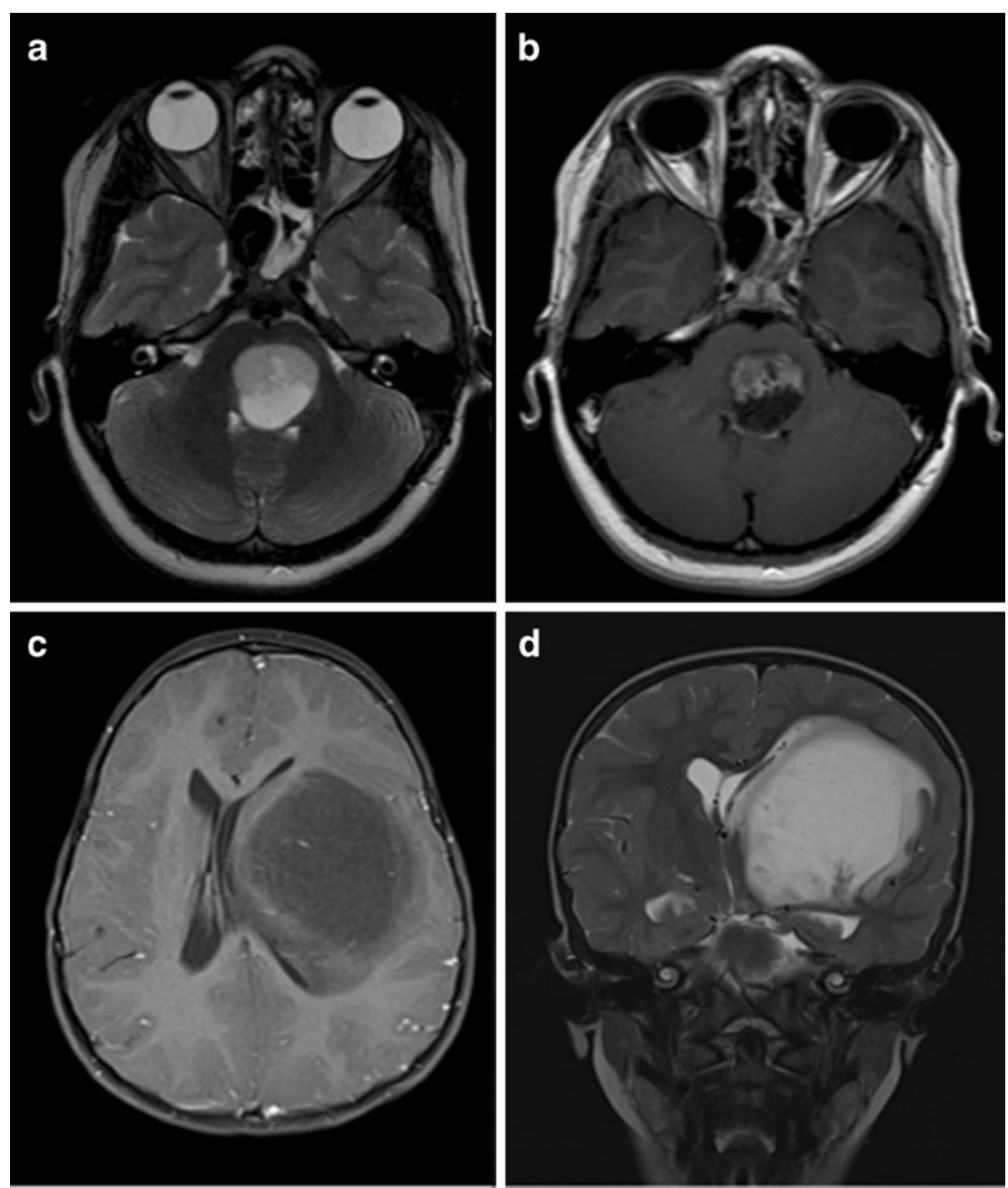
from glial tumors. Careful evaluation of the site of origin may distinguish an optic glioma from sellar tumors. The presence of calcifications and cysts may support the diagnosis of craniopharyngioma.

The definitive diagnosis is made with tissue evaluation if possible. If the tumor is accessible in a non-eloquent area, complete surgical resection may be the definitive treatment choice. Otherwise, a biopsy or subtotal resection may be possible to achieve the diagnosis and to relieve mass effect. In cases of optic pathway lesions, the diagnosis is often made based on the radiographic appearance because surgical manipulation can be unacceptably morbid. If the serum beta-HCG or alpha-fetoprotein is elevated, then a tissue diagnosis is not mandated if no other reason for marker elevation is found.

\section{Low-grade glioma subtypes}

As mentioned above, LGGs are a heterogeneous group of tumors with different cells of origin. The most common pediatric LGGs are of astrocytic origin. Tumors of neuroglial origin, such as gangliogliomas or dysembryoplastic neuroepithelial tumors, are less common and are typically managed with symptom management and/or surgery with good results. Oligodendroglial or mixed oligoastrocytic tumors are uncommon in children. LGGs are graded after assessment of the proliferative index, nuclear atypia, presence of microvascular proliferation, and necrosis. Astrocytomas are classified into four grades, and the classification scheme as well as the different pathologies is summarized in Fig. 2.

Pathologic diagnosis can be challenging if the biopsy sample is small or crushed. Tumor cells can be infiltrative through the normal brain, and identification of abnormal cells can be difficult. Immunohistochemistry stains for the following: synaptophysin, glial fibrillary acidic protein, keratin, p53 protein, S-100, and epithelial membrane antigen, can assist in tumor diagnosis. Cytogenetic markers such as IDH 1 mutation status and 1p19q deletion status are useful

\begin{tabular}{|c|c|c|c|c|c|}
\hline Astrocytic Tumors & $\mathbf{I}$ & II & III & IV & \multirow{3}{*}{$\begin{array}{l}\text { well } \\
\text { circumscribed }\end{array}$} \\
\hline Subependymal giant cell astrocytoma & $x$ & & & & \\
\hline Pilocytic astrocytoma & $\mathrm{x}$ & & & & \\
\hline Pilomyxoid astrocytoma & & $\mathrm{x}$ & & & \multirow{3}{*}{ cytologic atypia } \\
\hline Diffuse astrocytoma & & $x$ & & & \\
\hline Pleomorphic xanthoastrocytoma & & $x$ & & & \\
\hline Anaplastic astrocytoma & & & $x$ & & anaplasia, mitosis \\
\hline Glioblastoma & & & & $x$ & \multirow{3}{*}{$\begin{array}{l}\text { microvascular } \\
\text { prolif, necrosis }\end{array}$} \\
\hline Giant cell glioblastoma & & & & $x$ & \\
\hline Gliosarcoma & & & & $x$ & \\
\hline
\end{tabular}

Fig. 2 WHO classification of astrocytic tumors with characteristic pathologic findings in discriminating oligodendroglial tumors and appear to have prognostic significance.

In children, the most common LGG is pilocytic astrocytoma that is characterized by bipolar cells with long hair-like GFAP positive processes, Rosenthal fibers, eosinophilic granular bodies (EGBs) and microcysts. Grade 2 astrocytomas tend to be infiltrative which is seen in the pathologic specimen and on neuroimaging. Oligodendrogliomas may be associated with calcifications, have a "fried egg" appearance, have the $1 \mathrm{p} 19 \mathrm{q}$ codeletion, and may be more likely to involve the surface of the brain. Gangliogliomas are typically located in the medial temporal lobes and can be enhancing, and patients often present with seizure activity. They may have EGBs and have binucleate cells.

\section{Pilocytic astrocytoma}

PA constitute $16 \%$ of all CNS tumors diagnosed in patients under the age of 19 [1]. The typical presenting findings are due to the mass effect on the normal tissue around the site of origin. Eighty to eighty-five percent of cerebellar astrocytomas are PA. On imaging, these tumors are well circumscribed and may be solid or have cystic components. These tumors often enhance with contrast as shown in Fig. 1. Gnekow et al. reported that a group of 700 of 1,031 (68\%) patients enrolled on the HIT-LGG-1996 study had PA. Of these, 39, 37, 10, and $9 \%$ arose in the cerebellar hemisphere, midline supratentorial brain, brainstem, and cerebral hemisphere, respectively [4].

Management of PA depends on the age of the patient and the size and location of the tumor. If the tumor is found incidentally and is not associated with mass effect or symptoms, interval radiographic surveillance may be appropriate if the patient and family is able to return for routine evaluation. Biopsy or definitive resection may take place at the time of radiographic or clinical progression.

In patients with symptomatic disease, complete resection is associated with an $85 \%$ event-free survival rate and $90 \%$ overall survival rate up to 16 years [4]. For patients where recurrent disease is identified after initial surgery, a second resection may be considered. For patients with symptomatic, progressive, or recurrent disease that is deemed unresectable, treatment options depend on the age and NF1 status of the patient. If the patient is under the age of 10 years, chemotherapy has often been used to delay and/or avoid the use of radiotherapy until the patient has progression and is older. Ater et al. reported the outcomes of the Children's Oncology Group study (A9952) that evaluated carboplatin and vincristine $(\mathrm{CV})$ vs. thioguanine, procarbazine, lomustine, and vincristine (TPCV) in a randomized study on 274 non-NF1 patients (124 PA). No difference was noted between the two chemotherapy regimens on the planned data analysis in the 5year event-free survival or overall survival rates (45 and $86 \%$, 
respectively, for the whole group); however, TPCV appeared to be associated with a better 5-year event-free survival on a secondary unplanned analysis method (39 vs. $52 \%$ for CV and TPCV, respectively; $p=0.007)$. Salvage therapy after first failure is not reported on this study. The authors recommend considering either of these regimens with consideration of the associated toxicities [5]. In those patients that are found to have progressive disease after initial chemotherapy management, salvage interventions include second-line chemotherapy, radiotherapy (RT), or reconsideration of surgery.

Radiotherapy is still considered the definitive treatment for unresectable PA. The rationale for using chemotherapy in younger patients is to avoid the potential long-term effects of RT such as neuroendocrine, neurocognitive, audiologic, and visual changes or secondary malignancies. In children older than 10 years, it is felt that the risks of RT are lower; therefore, in non-NF1 patients with unresectable, progressive, and symptomatic disease, RT should be considered. Modern RT uses MRI planning to ensure appropriate tumor and normal tissue delineation. Conformal radiotherapy with photonbased techniques such as intensity-modulated radiotherapy, arc therapy (VMAT), tomotherapy, and proton therapy are all appropriate modalities. Proton therapy is particularly attractive in this vulnerable population because there is consistently a reduction in radiation dose to the normal brain and other normal tissues that are not abutting the tumor volume. Early results from Kahalley et al. are suggesting better preservation of neurocognitive function in a group of patients treated for CNS tumors treated with proton in comparison to a similar group who had been treated with photon radiation [6]. In the SIOP study, 147 patients (108 with PA) received RT (96 external beam, $51^{125}$ I brachytherapy) either at initial diagnosis (67/147) or after observation (80/147). The following response rates were reported: complete response $14 \%$, partial response $34 \%$, stable disease $43 \%$, and progressive disease $9 \%$. The best response was noted at a median of 6 months (range 1-37 months). At last follow-up, 136/147
(92\%) of the patients who received RT were alive, with 39 patients receiving multiple treatment approaches [4].

When patients are treated with any form of RT, parents and the patient should be informed on potential late toxicities, and these functions should be evaluated in a long-term fashion. The younger the patients, the more vulnerable they are to these side effects, and all efforts should be made to minimize these toxicities as much as possible when designing the radiotherapy plan.

Prognostic factors that impact overall survival and eventfree survival are complete resection which follows anatomical location since these two factors are related, pilocytic vs. nonpilocytic tumors, and age, with younger age being worse. It is becoming apparent that the biomolecular and genetic makeup of the pilocytic astrocytomas may vary between patients and between the different primary locations.

Patients with NF1 are at a higher risk of developing CNS tumors, particularly optic nerve gliomas (ONGs). If patients are found to have NF1 by clinical examination, a thorough ophthalmologic examination is warranted. If young patients are found to have an ONG, they should be evaluated for NF1. Since the natural history of ONGs in patients with NF1 can vary, observation with serial examinations (MRI and clinical) is reasonable. Once growth or visual decline is noted, then intervention is appropriate. Since these tumors are rarely resectable and RT is potentially associated with a higher risk of vascular injury, chemotherapy is advocated as the first approach, despite the age of the patient. Typically, ONGs associated with NF1 will be diagnosed before adolescence and often before the age of 5 years.

\section{Non-pilocytic gliomas}

Grade 2 astrocytomas have an infiltrative growth pattern, which renders them more challenging to obtain a complete resection (Fig. 3). In addition, the natural history of these
Fig. 3 Two examples of MRI scans of patients with grade 2 astrocytoma. a Axial fluidattenuated inversion recovery (FLAIR) MRI scan of large bithalamic glioma with associated mass effect and hydrocephalus. b Axial FLAIR MRI scan of left frontal diffuse glioma in patient who presented with seizure activity
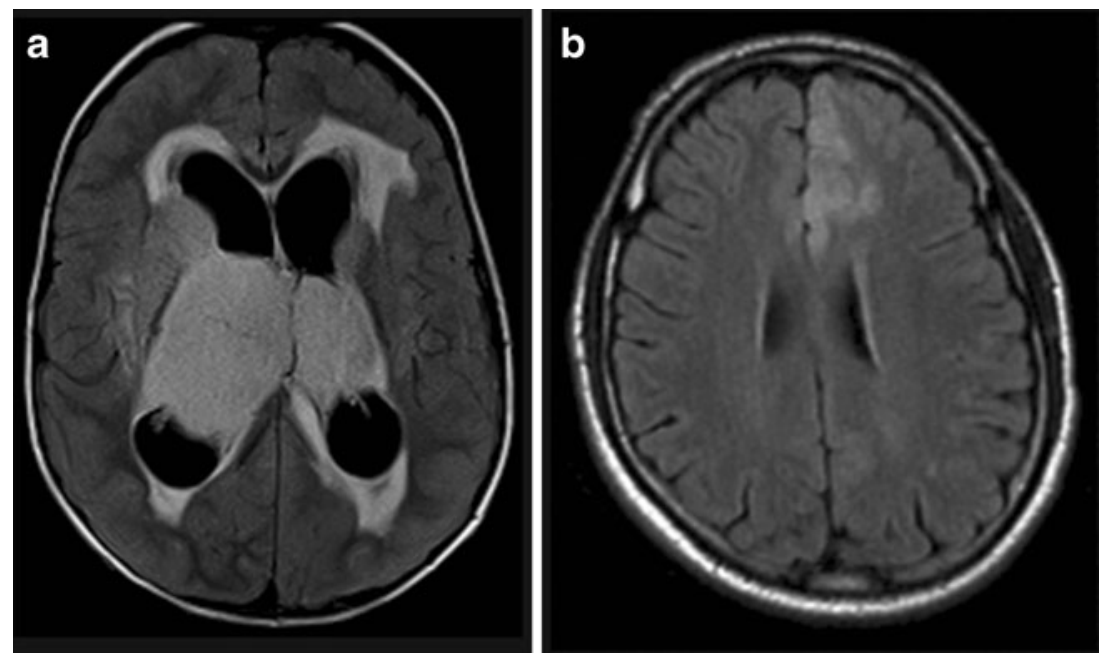
tumors is to dedifferentiate to higher grade gliomas over time. The diagnosis and management approach is similar to that of pilocytic astrocytoma, but the expectations are that these patients will have a worse outcome over time, 94 vs. $87 \% 10$-year overall survival rate $(p=0.04)$ and 50 vs. $40 \%$ 10 -year event-free survival rate $(p<0.001)$ [4].

Gangliogliomas are glioneuronal tumors that have a tendency to grow in the medial temporal lobes, and seizures are often a presenting symptom for many patients. Management in these patients includes medical management of the seizures, possible resection of the tumor, and seizure focus as definitive treatment. Local conformal RT can be considered in patients for progressive unresectable disease.

Oligodendroglioma is quite uncommon in children. In adults, the identification of the codeletion of $1 \mathrm{p} 19 \mathrm{q}$ chromosome arms has become integral in the patient assessment and treatment plan. In older patients, the presence of this codeletion is considered a hallmark of these tumors and confers a better response to both chemotherapy and radiotherapy in comparison to astrocytomas. In adults, if complete surgical resection is not possible, then chemotherapy with procarbazine, $\mathrm{CCNU}$, and vincristine or temozolomide is considered as the next step. RT is reserved for progressive disease or anaplastic oligodendroglioma.

\section{Summary}

Pediatric brain LGGs represent a heterogeneous group of tumors that require a multidisciplinary management for the diagnosis, management, and follow-up. Pilocytic astrocytoma is the most common subtype of LGG, and treatment options can vary from observation, surgery, chemotherapy, radiotherapy, or a combination of them. Long-term outcomes are reasonable, so long-term surveillance for recurrence and management of toxicities is mandatory. The treatment team should include a dedicated group of pediatric neurosurgeons, neuropathologists, neurooncologists, radiation oncologists, ophthalmologists, and endocrinologists to optimize the treatment approach and follow-up of these vulnerable patients.

Conflict of interest I do not have any conflicts of interest to declare with this work.

\section{References}

1. CBTRUS (2012) CBTRUS statistical report: primary brain and central nervous system tumors diagnosed in the United States in 2004-2008. CBTRUS, Hinsdale

2. WHO (2007) WHO classification of tumours of the central nervous system. IARC, Lyon

3. Forbes JA, Reig AS, Smith JG, Jermakowicz W, Tomycz L, Shay SD, Sun DA, Wushensky CA, Pearson MM (2011) Findings on preoperative brain MRI predict histopathology in children with cerebellar neoplasms. Pediatr Neurosurg 47(1):51-59. doi:10.1159/000329627

4. Gnekow AK, Falkenstein F, von Hornstein S, Zwiener I, Berkefeld S, Bison B, Warmuth-Metz M, Hernaiz Driever P, Soerensen N, Kortmann RD, Pietsch T, Faldum A (2012) Long-term follow-up of the multicenter, multidisciplinary treatment study HIT-LGG-1996 for low-grade glioma in children and adolescents of the German Speaking Society of Pediatric Oncology and Hematology. Neuro Oncol 14(10):1265-1284. doi:10.1093/neuonc/nos202

5. Ater JL, Zhou T, Holmes E, Mazewski CM, Booth TN, Freyer DR, Lazarus KH, Packer RJ, Prados M, Sposto R, Vezina G, Wisoff JH, Pollack IF (2012) Randomized study of two chemotherapy regimens for treatment of low-grade glioma in young children: a report from the Children's Oncology Group. J Clin Oncol 30(21):26412647. doi:10.1200/JCO.2011.36.6054

6. Kahalley LS, Ris MD, Moore BD, Okcu MF, Chintagumpala M, Grosshans DR, Paulino AC, Mehta PD, Stancel HH, Pipkins T, Mahajan A (2012) Change in IQ over time among pediatric brain tumor patients treated with proton beam radiation therapy versus conventional radiation therapy. Société Internationale d'Oncologie Pédiatrique, London 